\title{
A Review on Effectiveness of Real time optimization techniques in Power Management System in Hybrid Electric Vehicle
}

\author{
MANila VERMa, SWAPNIL SRIVASTAVA
}

\begin{abstract}
There is need of exploring the knowledge about energy management system which is used in HEV to enhance its performance and meet the demand of driver and vehicle also reduces pollution, fuel depletion and carbon emission. Also in order to provide better efficiency, power management system plays a important role in HEV operation. Several power management techniques are available for real time application and can provide better efficiency for different driving condition. Rule based methods and Optimization based methods focused here. In rule-based methods, methods are easily implemented and are very simple in real time but cannot provide best of optimality whereas in optimized based methods, it can provide better results but at high cost of high computational requirements and price. Thus, in this review- based paper we will brief about the latest trend of EMS and power system requirement that are based upon rule based and optimization based and will highlight on pros and cons. Another method is Pontryagin's Minimum Principle (PMP) which research the intent of the driver and the operation state identification of HEV based on Markov theory, in full consideration of the people-vehicle-road influence on power management system and problem of optimum solution in power management in case of variation of real operating problem is eliminated.
\end{abstract}

Index Terms- Hybrid electric vehicle(HEV), Energy Management System (EMS), Pontryagin's Minimum Principle (PMP), rule based and optimization based.

\section{INTRODUCTION}

In transportation, hybrid electric vehicles (HEV) represent a significant step towards reducing dependency on depleting fossil fuels. Hybrid vehicles are the ones with more than one type of power plant which supplies energy to the vehicle. The power distribution is needed during the driving operation, so the energy management strategy used that directly affects its performance. In public transportation system bus lines are relatively fixed and it starts and stops frequently. Hybrid bus can avoid frequent start and stop and reduce the idling driving condition of vehicle, so the study of energy management strategy can

Manila Verma, Department of Electrical Engineering, United College of Engineering and Research Prayagraj, INDIA

Swapnil Srivastava, Department of Electrical Engineering, United College of Engineering and Research, Prayagraj, INDIA obtain good control effect, carrying out relevant research has positive significance and practical application value. A mathematical problem is made with respect to input and outputs with power management constraints [1]-[5].

HEV consists of engine system, electric drive system, chassis, body, electric equipment and so on. Compared with traditional cars, the biggest difference between them is the driving system, secondly is the difference of the auxiliary system caused by different driving system. The driving system of HEV is composed of engine system, driver motor system, motor speed, control device, power source and so on, and the energy of motor comes from the battery.

\section{ENERGY MANAGEMENT PRINCIPLE AND POWER SYSTEM OF HEV}

HEV can be classified into two manners-

(1) Power-driven connection

(2) Degree of hybridization.

Diving mode can be divided into series, parallel, parallel-series and according to degree of hybridization it can be differentiate in micro hybrid, medium hybrid, strong hybrid and Plug-in. The schematic diagram of parallel-serial HEV power system is shown in Figure 1.

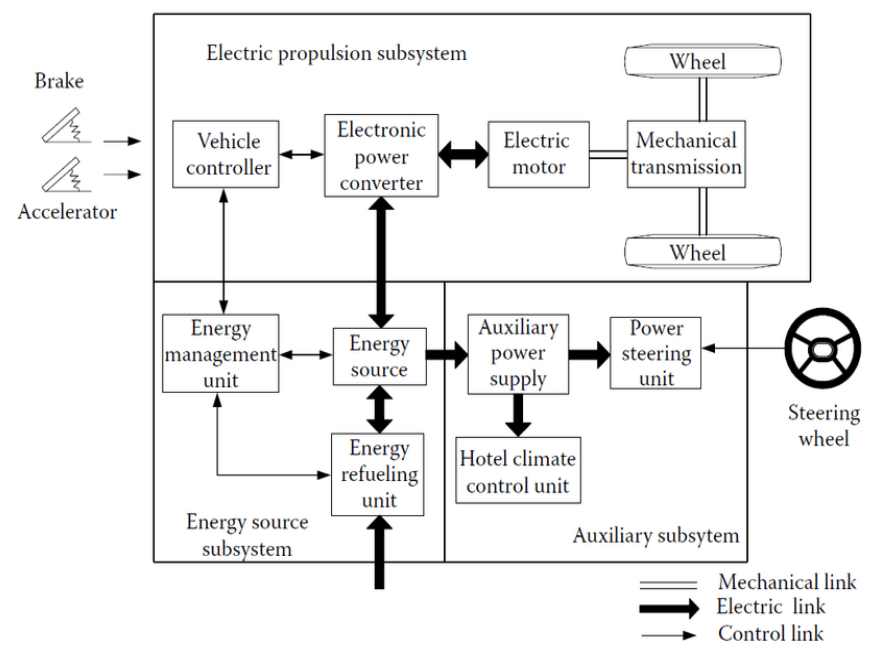

Fig.1 Schematic block diagram of HEV

According to the current driving intention, battery, motor and engine parameters, vehicle acceleration, deceleration, braking, battery pack temperature, voltage, current and 
SOC and the mathematical model of energy management problems such as performance index, state of SOC and power constraint is established. Traditional energy management control uses energy management control strategy to select the working mode and coordinate the work of each component.

\section{RESEARCH BASED ON REVIEW}

Energy management strategies are rules based and optimization based. Rule- based is to preset the logic rules like vehicle parameters and adjust the working state of each component of HEV to improve the whole performance of the vehicle [10]. This control algorithm is easy to implement and has good stability, and is the earliest control method used in HEV. The logic rules can be divided into deterministic rules and fuzzy logic rules. Because the essence of it depends on empirical rules, and the control accuracy cannot be guaranteed in practical application [11]-[14].

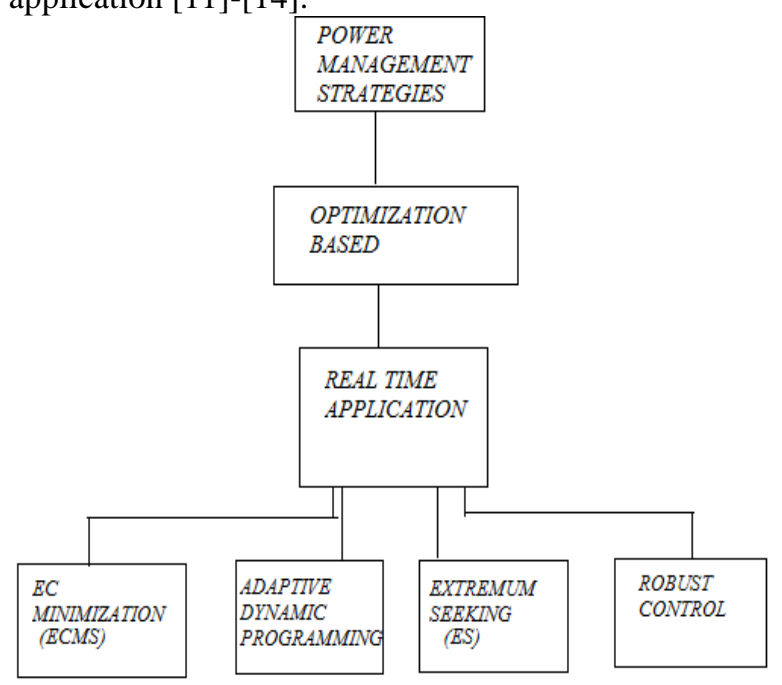

Fig.2 Categorization of HEV Control Strategies

Energy management strategy based on optimization can be divided into....

1) Equivalent Consumption Minimization Strategies (ECMS)

2) Adaptive Dynamic Programming (ADP)

3) Extremum Seeking (ES)

4) Robust Control (RC)

\section{A) $E C M S$}

ECMS (Equivalent cost minimization strategy) method used equal factors to transfer the electric energy depletion to fuel usage but the estimation of equivalent factors is critical. In the old model of ECMS, power train components have constant efficiencies but now it is dynamic.

ECMS control strategy is used under the condition of vehicle performance constraints and calculate engine fuel consumption and motor power consumption at every instantaneous moment of the vehicle driving process. Disadvantages of this method are multiple calculations needed, real-time performance is poor, and adaptability to dynamic variation is poor and lack of theoretical basis for the determination of control parameters [2]-[4]. Recently intelligent algorithms are used to the energy management strategy of $\mathrm{HEV}$, which can optimize and improve traditional energy management strategies, but cannot fundamentally avoid the possibility of getting into local optimal. Also, the introduction of intelligent algorithms increases the optimization time, it is more difficult to apply in the actual operation of vehicles.

\section{B) $A D P$}

It simplified the system by reducing the computational load time in a dynamic problem and reconstructed version provides a near suboptimal solution yet it only performs better as compared to many real time methods. Another method known as iterative DP is used to determine the optimal strategies for upcoming iterations by using coarse grids for control and state vectors. By progressive iterations the grids are purified to achieve the global optimal in view to save the fuel at less calculations and reduced memory requirements. It is simple but the knowledge of driving cycle is required, even the changes in the traffic are to be noticed [12].

\section{C) Extremum Seeking $(E S)$}

This method is not like one of classical control examples where formation doesn't have stability defined in it.

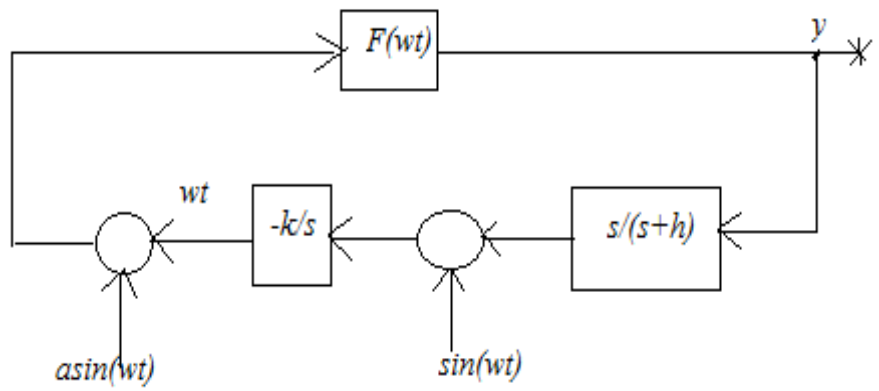

Fig.3 ES Scheme

Based on restoring unknown plants with the use of periodic excitation inputs and also with the use of the system output we can examine the gradient and also, we can find out the minima/maxima at zero gradient locations.

As the figure 3 shows the method uses the disturbed sinusoidal signal $(\omega t)$ to get $\mathrm{f}(\mathrm{wt})$ which is a nonlinear system to measure an output.With the help of filter output is filtered and operated with sin signal to determine the static gradient of the plant and integrator is used [21]-[24]

\section{D) Robust Control (RC)}

This method is applied to the system which has uncertainty, disturbing inputs, noisy measurement system to achieve stability and robustness in the system. Designed parameters provide desired outputs and sensitivity of the 
system output that will get attenuated from the many effects coming from the unknown inputs. In this method the depletion of SOC in terms of electric and chemical energy and the power consumed is defined. When the input is described as electric power, the minimization problem can be designed by maintaining the input near to the demanded power. The results of this method are still inferior to the ECMS or Dynamic Programming approach. The main disadvantages are problem complexity is high results in incapability to find an desired solution at the time of peak [30]-[35].

\section{E) PROBLEMS}

1) Contradiction between real-time and global optimum of energy management strategy of $\mathrm{HEV}$ control strategies.

2) Power management strategy is depend upon fuel consumption, carbon emission and driving cycle but control parameters are adjusted by actual working conditions to achieve global optimal control so as to improve the real-time and practicability of energy management strategy.

3) The vehicle demand power is calculated according to actual working conditions, bus parameters and power train model so that the control strategy cannot achieve global optimal of energy management under the dynamic changes of the actual working conditions.

\section{KEY TECHNOLOGY OF ENERGY MANAGEMET IN HEV}

\section{A) Energy management strategy based on PMP}

Russian mathematician "Pontryagin" and his students deduced the optimal control theory named Pontryagin's Minimum Principle in the 1950s; this principle has been widely used in various fields, and has been applied to the energy management strategy of HEV in the early 2000s. In order to make fuel economy performance of HEV reach the optimum value, scholars use Pontryagin's Minimum Principle (PMP) as the theoretical basis and manage the energy of HEV in a way that provides the necessary conditions for optimization in real time. This method has less calculation compared with DP and is suitable for real-time control [30]. PMP is a kind of local optimization theory, while it can get global optimization results under certain conditions.

Based on PMP, global optimization is discussed to solve the drawback that traditional global optimization cannot be applied to reality. The selection of control parameters in PMP is another key problem in the application of energy management. The power distribution results between power sources are directly affected by control parameters, which would affect the ultimate power performance and economy. Determining factors affecting the initial value of control parameters are analyzed, and then the method of determining control parameters is proposed.
Using the sum of fuel consumption at each moment as the performance index, using SOC of the battery as system the state variable and output power of generator set and battery pack as constraint conditions under the condition of keeping the battery balance, Vehicle demand power is corrected in real time and calculated power distributed between generator set and battery pack. The optimal solution is obtained using PMP, and the optimal control of energy management of hybrid buses is realized [25].

\section{CONCLUSION}

An energy management and supervisory control system (EMS) for a hybrid electric vehicle (HEV) is an essential component of HEV. In this paper Energy transfer mechanism of hybrid vehicle is deeply studied, and which can adapt for driving intention. Also the traditional energy management strategies are explained here. It provides a good theoretical basis for the key technology of HEV in China, Russia and it provides new technical aspects for energy management problems of $\mathrm{HEV}$, and has massive technical significance. It is also observed that two major techniques are used for controlling the HEV operation rule based method and optimization based method. In this paper gave introduction to the new innovative trends in this field. For each method there were advantages and disadvantages highlighted. The optimization-based methods, real time techniques are extensive as it provides the nearest global optimal solution.

\section{ACKNOWLEDGMENT}

I thank to my colleagues from United College of Engineering and Research, Prayagraj for giving their valuable knowledge, insights and their expertise which greatly improve the research on $\mathrm{HEV}$.

I want to give special thanks to Mr. Swapnil Sivastaca, Associate Professor EED for assistance with the understanding of different optimization techniques and his support.

\section{REFERENCES}

[1] M. Ali, Ahmed \& Söffker, D (2018). Towards Optimal Power Management of Hybrid Electric Vehicles in Real-Time: A Review on Methods, Challenges, and State-Of-The-Art Solutions. Energies. 11. 10.3390/en11030476.

[2]B. Bose, J. Shah, "Simulation based V2H Super Capacitor Charging using Bidirectional DC-DC Converter," 2018 International Conference on Smart City and Emerging Technology (ICSCET), Mumbai, 2018, pp. 1-5.

[3] Navale and T. C. Havens, "Fuzzy logic controller for energy management of power split hybrid electrical vehicle transmission," 2014 IEEE International Conference on Fuzzy Systems (FUZZ-IEEE), Beijing, 2014, pp. 940-947

[4] S. A. Zulkifli, S. Mohd, N. Saad and A. R. A. Aziz, "Influence of Motor Power and Efficiency on Fuel Consumption of RetrofitConversion Split-Parallel Hybrid Electric Vehicle," 2013 IEEE Vehicle Power and Propulsion Conference (VPPC), Beijing, 2013, pp. 1-5.

[5] Y. Zeng, "Research on the parameter matching and CVT's target speed ratio optimization of plug in hybrid electric vehicle*," 2017 
[6]Dam Hoang Phuc, Pongsathorn Raksincharoensak, Masao Nagai and Masahiro Suzuki, "Control Strategy for Hybrid Electric Vehicles Based on Driver Vehicle Following Model" SICE-ICASE International Joint Conference 2006 Otc.18-21, 2006 in Bexco, Busan, Korea

[7] Fazal U. Syed, Ming L. Kuang, Matt Smith, Shunsuke Okubo, "Fuzzy Gain-Scheduling Proportional-Integral Control for Improving Engine Power andSpeed Behavior in a Hybrid Electric Vehicle" IEEE transactions on vehicular technology, vol. 58, no. 1 , january 2009

[8] Liu Qingkai, Zhao Zhiguo, Dai Haifeng(2009) "Energy Management Strategy for single drive shaft parallel hybrid electric vehicle based on torque control" 978-1-4244-2601-0/09/\$25.00 @2009 IEEE.

[9] Rüdiger Appunn and Kay Hameyer , "Simulation of a High Voltage System in a Hybrid Electrical Vehicle" 2010, 12th International Conference on Optimization of Electrical and Electronic Equipment, OPTIM 2010

[10] Yun Zhang, Mingshuang Bi, Xiumin Yu, Sina Xu, "An Adaptive Neural PID Controller for Torque Control of Hybrid Electric Vehicle", in 6th International Conference on Computer Science \& Education (ICCSE 2011) August 3-5, 2011. SuperStar Virgo, Singapore.

[11] Hicham Fakham, Di Lu, Bruno Francois, "Power Control Design of a battery charger in a Hybrid Active PV generator for load following applications" IEEE Transaction on Industrial Electronics, Vol. 58, Iss. 1 , pp. 85-94, Jan. 2011, TIE-09-1370

[12] Shijing Xu, "Investigation of EMS based on fuzzy logic controller for an ICE/battery/UC hybrid electric vehicle" 978-1-4577-0536-6/11/\$26.00 @2011 IEEE

[13] R A Hanifah , S F Toha , S Ahmad (2013) “PID-Ant Colony Optimization (ACO) control for Electric Power Assist Steering system for electric vehicle" Proc. of the IEEE International Conference on Smart Instrumentation, Measurement and Applications (ICSIMA) 26-27 November 2013, Kuala Lumpur, Malaysia

[14] Daniel Sigmund, Dipl.-Ing. Magnus (2014), "Simulation based development of an Energy Management System for a drive train of a Parallel Hybrid Electric Vehicle" 16th International Power Electronics and Motion Control Conference and Exposition.

[15] Sreenath M, Indulal S, "Design and Analysis of a Fuzzy Logic Controller for the Optimal Switching of Drives for Hybrid Electric Vehicles" 2015 International Conference on Control, Communication \& Computing India (ICCC) | 19-21 November 2015 | Trivandrum

[16] Yugong Luo, Tao Chen, Shuwei Zhang, and Keqiang Li, "Intelligent Hybrid Electric Vehicle ACC With Coordinated Control of Tracking Ability, Fuel Economy, and Ride Comfort" IEEE transactions on intelligent transportation systems 1524-9050 @ 2015 IEEE

[17] Mohamed yaich, mohamed radhouan hachicha, moez ghariani, "Modeling and Simulation of Electric and Hybrid electric vehicle for Recreational vehicle" 16th international conference on Sciences and Techniques of Automatic control \& computer engineering - STA'2015, Monastir, Tunisia, December 21-23, 2015

[18] Hamed Elwarfalli, Akram Muntaser, Jitendra Kumar, "Design and Implementation of PI Controller for the Hybrid Energy System" 978-1-5090-3441-3/16/\$31.00 (2016 IEEE

[19] Muhammad Ahsan saeed, Nisar Ahmed, Mujahid (2016) "A Comparative Study of Controllers for Optimal Speed Control of Hybrid Electric Vehicle" 978-1-4673-8753-8/16/\$31.00 @2016 IEEES

[20] Li, K. H. Nam, "Design and analysis of a DC-DC converter for EVs battery charger", IEEE 7th International Power Electronics and Motion Control Conference Asia, 2012, Harbin China

[21] G. Livinl, V. Horga, M. Raloi, M. Albu, Control of Hybrid Electrical Vehicle, John Wiley and Sons. Ltd., 2006

[22] Palwinder Kaur, Vikas Kumar, Richa Sharma (2016) "Speed Control of Hybrid Electric Vehicle using PSO based Fractional Order PID controller" 978-1-4673-6984-8/16/\$31.00 @ 2016 IEEE

[23] Cao J, Emadi A. "A new battery/ultracapacitor hybrid energy storage system for electric, hybrid, and plug-in hybrid electric vehicles", IEEE Transactions on power electronics. 2012 Jan; 27(1):122-32

[24] J. L. Torres, R. Gonzalez, A. Gimenez, and J. Lopez, "Energy management strategy for plug-in hybrid electric vehicles. A comparative study,” Appl. Energy, vol. 113, pp. 816-824, Jan. 2014.

[25] Mochammad Rameli, Yosi Riduas Hais, Rusdhianto Efendi Abdul Kadir, "Design of Self Commutation BLDC Motor with Torque Control Strategy Using Fuzzy Logic in Hybrid Electric Vehicle (HEV)" 2017 International Seminar on Intelligent Technology and Its Application

[26] Amirtha lakshmi, Veera raghavan, Ajinkya Bhave, "Driving Scenario Recognition for Advanced Hybrid Electric Vehicle Control" 2017 IEEE Transportation Electrification Conference (ITEC-India)

[27] Araz Saleki, Saman Rezazade, Mahmudreza Changizian, "Analysis and Simulation of Hybrid Electric Vehicles for Sedan Vehicle" 25th Iranian Conference on Electrical Engineering (ICEE2017)

[28] Namwook Kim, Sukwon Cha, Huei Peng, "Optimal Control of Hybrid Electric Vehicles Based on Pontryagin's Minimum Principle"

[29] Zhenhua Cai, Beigao Chen, Chengxin Luo, “A Fuzzy control based energy management strategy for LFP/UC hybrid electric vehicular energy system" 978-1-5386-1127-2/17/\$31.00 @2017 IEEE

[30] Sabah Saib, Zahir Hamouda, "Energy Management in a Fuel Cel Hybrid Electric Vehicle using a Fuzzy Logic Approach" The 5th International Conference on Electrical Engineering - Boumerdes (ICEE-B) October 29-31, 2017, Boumerdes, Algeria.

[31] Kai Han, Jili Tao, Zhang Ridong, Zhou Huiyu (2018) "PFC and Fuzzy based PI controller for bidirectional DC/DC converter of Buck mode in HEV" 978-1-5386-1243-9/18/\$31.00 \&2018 IEEE

[32] Raghavaiah Katuri, Srinivasa Rao Gorantla, "Design of a new controller for switching of energy sources applied to Hybrid Electric vehicle" International Conference on Recent Trends in Advanced Computing (ICRTAC-CPS 2018)

[33] Jinglai Wu , Jiageng Ruan, Nong Zhang, and Paul D Walker(2019) "An Optimized Real-Time Energy Management Strategy for the Power-Split Hybrid Electric Vehicles" IEEE transactions on control systems technology

[34] Abhishek Gaurav, Anurag Gaur (2020) "Modeling of Hybrid Electric Vehicle Charger and Study the Simulation Results" 978-1-7281-7549-2/20/\$31.00 @2020 IEEE

[35] Mehrdad Ahsani, Yimin Gao and Ali Emadi, "Modern electric, Hybrid Electric and Fuel cell Vehicles" (O2005 CRC press L 